WU-AP/07/90

UTAP-107/90

FERMILAB-PUB-90/52-A

\title{
Soft Inflation
}

ANDREW L. BERKIN ${ }^{1,(a)}$, KeI-ICHI MAEDA ${ }^{1,3,(b)}$ and JUN'ICHI YOKOYAMA ${ }^{2,3,(c)}$

1 Department of Physics, Waseda University, Shinjuku-ku, Tokyo 169, Japan

${ }^{2}$ Department of Physics, Faculty of Sciences, The University of Tokyo, Tokyo 113, Japan and

${ }^{3}$ NASA/Fermilab Astrophysics Center

Fermi National Accelerator Laboratory, Batavia, IL 60510

\begin{abstract}
We study the cosmology resulting from two coupled scalar fields, one which is either a new inflation or chaotic type inflaton and the other which has an exponentially decaying potential. Such a potential may appear in the conformally transformed frame of generalized Einstein theories like the Jordan-Brans-Dicke theory. The constraints necessary for successful inflation are examined. We find conventional GUT models such as $\mathrm{SU}(5)$ are compatible with new inflation, while restrictions on the self-coupling constant are significantly loosened for chaotic inflation.
\end{abstract}

April, 1990

(a) BITNET: berkin@jpnwas00

(b) BITNET: maeda@jpnwas00

(c) DECNET: tkyvax::yokoyama 
The inflationary cosmology[1] purports to solve many longstanding cosmological problems, and has been the subject of much investigation during the previous decade[2]. However, there is no fully satisfactory model for the source of inflation yet. In this letter, we consider a matter content described by two coupled scalar fields, one of which has a decaying potential and the other which serves as the inflaton driving the expansion. In this theory, the constraints placed upon previous models are considerably loosened.

Inflation with two scalar fields has been considered before, with effects such as consequent [3] and double[4] inflation arising. The extended inflationary scenario[5] uses the Jordan-BransDicke (JBD) scalar[6] to achieve expansion which is slower than exponential. Applied to the original old inflationary scenario, a successful completion of the inflationary phase may be possible. The chaotic inflation model in the JBD theory has also been investigated[7].

The potential which will be discussed here arises in the following two cases: (i) Some supergravity or superstring models produce an exponential potential coupled to other scalar fields [8]. (ii) In the generalized Einstein theories, which are conformally equivalent to standard Einstein gravity with a scalar field, the potential of a standard scalar field is modified to obtain such coupling in the conformal frame[9]. The generalized Einstein theories include the JBD theory, induced gravity[10], any theory with non-minimal coupling, the curvature squared theory and effective four-dimensional theories arising from a higher-dimensional unified theory. In such cases we should also discuss what happens in the original physical frame. Decaying potentials have recently been considered by Peebles and Ratra[11] to account for the dark matter in the universe.

The decaying exponential potential in our model produces the same effect as the JBD scalar in the extended inflation scenario, to reduce the rate of expansion. As the inflaton rolls down a flat plateau, the other scalar field evolves on the exponential potential, resulting in power-law inflation[12,13]. Although we find weaker inflation than in the conventional new and chaotic models, we obtain a longer period of inflation due to this coupling and the much 
smaller potential energy at the end of inflation. Naively speaking, since the energy scale at the period of horizon crossing is related to density perturbations, we expect much weaker constraints on the parameters of inflation. When the inflaton is of new inflation type, the fine tuning of initial conditions is lessened and density perturbations are suppressed. For a chaotic type field, the restrictions placed upon the coupling parameter by density perturbations are reduced considerably. We will investigate whether the Coleman-Weinberg potential in the standard GUT model yields successful new inflation and what restrictions on the coupling $\lambda$ are necessary for successful chaotic inflation.

We use as our action

$$
S=\int d^{4} x \sqrt{-g}\left[\frac{1}{2 \kappa^{2}} R-\frac{1}{2}(\nabla \phi)^{2}-\frac{1}{2}(\nabla \psi)^{2}-e^{-\beta \kappa \phi} V(\psi)\right]
$$

where $\kappa^{2}=8 \pi G, V(\psi)$ is the inflaton potential and $\beta$ is a dimensionless coupling constant, which must be smaller than $\sqrt{2}$ to guarantee power-law inflation. Although presently known supergravity models yield $\beta \geq \sqrt{2}$, we may realize power-law inflation with the help of viscosity due to particle production[13]. Furthermore, it is possible in the generalized Einstein. theories to have small enough $\beta$, e.g. $\beta \equiv 2(\omega+3 / 2)^{-1 / 2}$ for the JBD theory, with the JBD parameter $\boldsymbol{\omega}>1 / 2[9]$.

Working in the spatially flat Friedmann-Robertson-Walker metric with cosmic scale factor $a(t)$, we find from the field equations:

$$
\begin{aligned}
& \ddot{\phi}+3 H \dot{\phi}-\beta \kappa e^{-\beta \kappa \phi} V(\psi)=0 \\
& \ddot{\psi}+3 H \dot{\psi}+V^{\prime}(\psi) e^{-\beta \kappa \phi}=0 \\
& H^{2}=\frac{\kappa^{2}}{3}\left[\frac{1}{2} \dot{\phi}^{2}+\frac{1}{2} \dot{\psi}^{2}+V(\psi) e^{-\beta \kappa \phi}\right]
\end{aligned}
$$

where $H=\dot{a} / a$ is the Hubble parameter, an overdot denotes time derivation, and a prime denotes differentiation with respect to the argument of the function.

Inflation occurs as $\psi$ slowly rolls down the potential $V(\psi)$ with its amplitude further decreasing as $e^{-\beta \kappa \phi}$. During this period, $\bar{\psi}$ and $\dot{\psi}^{2} / 2$ can be neglected in (3) and (4), 
respectively. If $\beta^{2} \ll 6$, this ansatz is true until $\psi$ rolls down into the field-oscillation region. We then find a unique attractor of the power-law inflationary solution [13]:

$$
\begin{aligned}
\kappa \phi & =\kappa \phi_{c}+\frac{2}{\beta} \ln (t / \kappa), \\
a & =a_{0}\left(t / t_{0}\right)^{2 / \beta^{2}}, \\
f(\psi) & =f\left(\psi_{0}\right)-\left(1-\beta^{2} / 6\right) \ln \left(a / a_{0}\right),
\end{aligned}
$$

where

$$
\exp \left[\beta \kappa \phi_{c}\right] \equiv \frac{\beta^{4} \kappa^{4} V(\psi)}{12\left(1-\beta^{2} / 6\right)}, \quad f(\psi) \equiv \kappa^{2} \int d \psi \frac{V}{V^{\prime}},
$$

and the subscript 0 denotes the value at $t_{0}$ when the universe enters the inflationary phase. While the initial value of $\phi$ may be arbitrary, the spacetime evolves into the above power-law inflationary phase in a few expansion times, since the above solution is the unique attractor. Thus, any value of $\phi$ approaches $\phi_{0} \equiv \phi_{c}+2(\beta \kappa)^{-1} \ln \left(t_{0} / \kappa\right)$ at $t_{0}$.

We consider the three constraints imposed by the horizon problem, density perturbations and the reheating temperature, both with a new inflation type Coleman-Weinberg potential[14], and a chaotic type potential. The former may be approximated for small $\psi$ as [2]

$$
V(\psi)=V_{0}-\frac{\lambda}{4} \psi^{4}
$$

where $V_{0}$ is the GUT scale and $\lambda \sim 1 / 2$ for the $\mathrm{SU}(5)$ model, although $\lambda$ depends weakly on $V_{0}$ as well. For the latter we consider

$$
V(\psi)=\frac{1}{n} \lambda_{n} \psi^{n}
$$

with $n$ an even integer and $\lambda_{n}$ arbitrary for now. A main problem of ordinary chaotic inflation is the fine-tuned small value of the coupling parameter imposed by density perturbations[1], in particular $\lambda_{4} \lesssim 10^{-1 \text { " }}$ for the quartic case.

When the scalar field $\psi$ evolves into the oscillation phase, inflation will end. Hence the condition $|\ddot{\psi} / 3 H \dot{\psi}|_{f} \sim 1$, or equivalently $\left|(\ln V)^{\prime \prime}\right|_{f} \sim 3 \kappa^{2}\left(1-\beta^{2} / 6\right)^{-1}$, fixes the value of $\psi_{f}$, 
where a subscript $f$ denotes the value at the end of inflation. Then we find

$$
\begin{array}{lll}
f(\psi)=\kappa^{2} V_{0} / 2 \lambda \psi^{2} & \text { and } & \kappa \psi_{f}=\left[\kappa^{4} V_{0} / \lambda\left(1-\beta^{2} / 6\right)\right]^{1 / 2} \text { for new inflation } \\
f(\psi)=\kappa^{2} \psi^{2} / 2 n & \text { and } & \kappa \psi_{f}=\left[n\left(1-\beta^{2} / 6\right) / 3\right]^{1 / 2} \text { for chaotic inflation }
\end{array}
$$

The horizon problem will be solved if[12]

$$
\left(\frac{a_{f}}{a_{0}}\right)^{1-\beta^{2} / 2} \gtrsim 10^{30} \frac{T_{R H, \text { max }}}{m_{P L}}
$$

where $m_{P L}$ is the Planck mass and $T_{R H, \max }$ is the maximal possible reheating temperature, given by $H_{f}^{2}=\kappa^{2} \pi^{2} g_{*} T_{R H, \text { max }}^{4} / 90$, with $g_{*}(T) \sim 100$ the effective number of particle species.

Another constraint comes from density perturbations. In particular, results from the cosmic microwave background radiation imply that $\delta \rho / \rho<10^{-4}$, where $\delta \rho$ is the perturbation in the density $\rho$. Generalizing the result of Lucchin and Matarrese[12], the amplitude of density fluctuations of an arbitrary scale at the epoch when that scale re-enters the Hubble horizon may be calculated as follows:

$$
\left.\frac{\delta \rho}{\rho} \sim H^{2} \frac{\max \{|\dot{\phi}|,|\dot{\psi}|\}}{\dot{\phi}^{2}+\dot{\psi}^{2}}\right|_{h} \sim \begin{cases}\left.\kappa^{2} H_{0} \frac{V}{|V| 1}\right|_{h}\left(1-\beta^{2} / 6\right)^{-1}\left(a_{h} / a_{0}\right)^{-\beta^{2} / 2} & \text { for }|\dot{\psi}|_{h}>|\dot{\phi}|_{h} \\ \kappa H_{0} \beta^{-1}\left(a_{h} / a_{0}\right)^{-\beta^{2} / 2} & \text { for }|\dot{\psi}|_{h}<|\dot{\phi}|_{h}\end{cases}
$$

where a value with subscript $h$ is evaluated at the time the perturbation originally left the horizon, and $\alpha_{f / h} \equiv \ln \left(a_{f} / a_{h}\right)$ is $\sim 50-70$ for scales currently entering the horizon. Because of the factor $\left(a_{h} / a_{0}\right)^{-\beta^{2} / 2}$ in (14), we expect much smaller density perturbations than those in the conventional model, loosening the parameter constraints.

If we account for the observed baryon asymmetry in terms of the standard baryogenesis mechanism[15] through heavy Higgs bosons, the reheating temperature should be larger than $10^{10} \mathrm{GeV}[16]$. For efficient reheating, we find

$$
T_{R H, \max }=T_{R H, \text { conv }} e^{-\beta \kappa \phi_{0} / 4}\left(\frac{a_{f}}{a_{0}}\right)^{-\beta^{2} / 4}
$$

where $T_{R H, \text { conv }} \equiv\left(30 V\left(\psi_{f}\right) / \pi^{2} g_{*}\right)^{1 / 4}$ is the maximum reheating temperature possible in the 
corresponding conventional model. We expect a lower reheating temperature than in the conventional model, although this depends on the detail of each reheating mechanism.

The three constraints, (13),(14) and (15), restrict the initial values $\psi_{0}$ and $\phi_{0}$, the coupling parameter $\beta$, and parameters in the potential $V(\psi)$. We next examine the permitted values for both new and chaotic inflationary potentials.

(i) New inflation type

From the above constraints, we obtain three conditions for the initial value $\psi_{0}$ :

$$
\begin{aligned}
& \psi_{0}<\psi_{c r, H} \equiv 0.16 H_{0} \lambda^{-1 / 2} e^{\beta \kappa \phi_{0} / 2}\left[1+0.017 \ln \left(V_{0}^{1 / 4} / 10^{15} \mathrm{GeV}\right)\right]^{-1 / 2} \\
& \text { (horizon problem) } \\
& \psi_{0}>\psi_{c r, R H} \equiv 0.19 H_{0} \lambda^{-1 / 2} \beta e^{\beta \kappa \phi_{0} / 2}\left[1+0.094 \ln \left(V_{0}^{1 / 4} / 10^{15} \mathrm{GeV}\right)\right]^{-1 / 2} \text { (reheating) } \\
& \psi_{0}<\psi_{c r, D} \equiv 0.15 H_{0} \lambda^{-1 / 2}\left(\alpha_{f / h} / 70\right)^{-1 / 2} e^{\beta \kappa \phi_{0} / 2} \\
& \times\left\{1-0.20 \beta^{-2}\left(\alpha_{f / h} / 70\right)^{-1}\left[1+0.14 \ln \beta-0.29 \ln \left(V_{0}^{1 / 4} / 10^{15} \mathrm{GeV}\right)\right]\right\}^{-1 / 2} \\
& \text { (density perturbations) }
\end{aligned}
$$

Note that $|\dot{\phi}|_{h}>|\dot{\psi}|_{h}$ unless $\beta \lesssim 10^{-10} \lambda^{-1 / 2}\left(\alpha_{f / h} / 70\right)^{-3 / 2}\left(V_{0}^{1 / 4} / 10^{15} \mathrm{GeV}\right)^{2}$. Imposing $\min \left\{\psi_{c r, H}, \psi_{c r, D}\right\}>\psi_{c r, R H}$, we find that $\beta<0.79\left(\alpha_{f / h} / 70\right)^{-1 / 2}$. If the condition $\lambda \lesssim 0.02 e^{\beta_{\kappa} \phi_{0}}$ is satisfied, the natural initial condition of $\psi_{0} \gtrsim H_{0}$ is allowed. These values are around the natural ones of unity, without need for fine-tuning. The constraints on $\lambda$ and $V_{0}$ are plotted together in figure 1 for $\beta=0.1, \psi_{0}=H_{0}$ and $\phi_{0}=10 m_{P L}$. There is a significant region in $\lambda-V_{0}$ space where all the constraints are satisfied. The $\mathrm{SU}(5)$ model with $V_{0}^{1 / 4}=10^{15} \mathrm{GeV}$ and $\lambda \sim 1 / 2$ is inside the allowed region. With this scenario, the slower expansion rate during inflation allows for the suppression of density fluctuations, which had been the main drawback of the regular new inflationary model. 
(ii) Chaotic inflation type

Assuming the initial energy scale is the Planck one, we obtain the constraints:

$$
\begin{aligned}
& \psi_{0}>\psi_{c r, H} \equiv 2.3 m_{P L} n^{1 / 2} \quad \text { (horizon problem) } \\
& \psi_{0}<\psi_{c r, R H} \equiv 2.6 m_{P L} n^{1 / 2} \beta^{-1} \quad \text { (reheating) } \\
& \psi_{0}>\psi_{c r, D} \equiv \begin{cases}1.5 m_{P L} n^{1 / 2} \beta^{-1}\left[1+2.5 \beta^{2}\left(\alpha_{f / h} / 70\right)\right]^{1 / 2} & \text { for } \beta<\left(n / 2 \alpha_{f / h}\right)^{1 / 2} \\
1.4 m_{P L} n^{1 / 2} \beta^{-1}\left[1+2.9 \beta^{2}\left(\alpha_{f / h} / 70\right)\right]^{1 / 2} & \text { for } \beta>\left(n / 2 \alpha_{f / h}\right)^{1 / 2}\end{cases}
\end{aligned}
$$

(density perturbations)

where $\beta \lesseqgtr\left(n / 2 \alpha_{f / h}\right)^{1 / 2}$ corresponds to $|\dot{\phi}|_{h} \lesseqgtr|\dot{\psi}|_{h}$, respectively. Just as in the new inflation model, we find $\beta<0.9\left(\alpha_{f / h} / 70\right)^{-1 / 2}$. Most significantly, much lower values of $\psi_{0}$ than in the standard case are now possible, leading to considerably larger and hence less fine-tuned values of the coupling constant $\lambda_{n}$. For example, $\lambda_{4}<5.8 \times 10^{-3} \beta^{4} e^{\beta \kappa \phi_{0}}$ for the quartic case and $\lambda_{2}<0.15 m_{P L}^{2} \beta^{2} e^{\beta \kappa \phi_{0}}$ for the massive scalar field, so we find a natural coupling constant $\lambda_{n}$ is possible. A different mechanism to obtain a similar constraint on $\lambda_{4}$ has been discussed by use of a non-minimal coupling term with $\xi<-2 \times 10^{4}$ [17].

If our model is derived from the generalized Einstein theories via a conformal transformation, we must transform back to the original system. We present the JBD model as one example. The JBD scalar field $\Phi_{\text {JBD }}$ is related to our $\phi$ via the conformal transformation $g_{\mu \nu}=e^{\beta \kappa \phi / 2} g_{\mu \nu}^{\mathrm{JBD}}$ as

$$
\kappa \phi=(\omega+3 / 2)^{1 / 2} \ln \left(\Phi_{\mathrm{JBD}} G\right)+\kappa \phi_{\text {present }}
$$

with $\beta=2(\omega+3 / 2)^{-1 / 2}[9]$. Since the JBD parameter $\omega$ is constrained by observations to be larger than $500[18], \beta$ is smaller than 0.09 in this model. The cosmic time $t_{\mathrm{JBD}}$ and the scale factor $a_{\mathrm{JBD}}$ are given by $d t_{\mathrm{JBD}}=e^{-\beta \kappa \phi / 4} d t$ and $a_{\mathrm{JBD}}=e^{-\beta \kappa \phi / 4} a$. Hence we obtain the formula[19]:

$$
\begin{aligned}
\left(a_{f} / a_{0}\right)_{\mathrm{JBD}} & \sim e^{-\beta \kappa\left(\phi_{f}-\phi_{0}\right) / 4}\left(a_{f} / a_{0}\right) \sim\left(a_{f} / a_{0}\right)^{1-\beta^{2} / 4}\left(V_{0} / V_{f}\right)^{1 / 4} \\
\left.\frac{\delta \rho}{\rho}\right|_{\mathrm{JBD}} & \sim \frac{3}{2} \frac{\delta \rho}{\rho}
\end{aligned}
$$




$$
T_{R H, \max }^{\mathrm{JBD}} \sim e^{\beta \ltimes \phi / 4} T_{R H, \max } \sim T_{R H, \text { conv }}
$$

Therefore, in the original system, the constraints from the horizon problem and density perturbations do not change much, while the reheating temperature is the same as in the conventional model, where we have assumed instantaneous reheating. Hence the constraint from successful reheating is loosened in the JBD theory (see Fig.1).

We have presented a scenario in which an inflaton potential coupled to an exponentially decaying potential leads to a less rapidly expanding inflationary stage. Hence, the problem of excessive density perturbations in the new inflationary model is removed and fine-tuning in the chaotic model is lessened. In addition, the less rapid expansion of the present model may allow successful old inflation, just as with extended inflation.

Finally, we mention that if the inflaton potential $V$ is nonzero at the termination of inflation, then a decaying cosmological constant will exist. Though primordial nucleosynthesis sets a stringent bound on the vacuum energy at that epoch [20], it may still be possible that the relic vacuum energy contributes significantly enough to the present total energy density to close the universe. Work on this possibility is in progress.

\section{Acknowledgements}

KM would like to thank J. Hwang at Univ. of Texas for useful discussions, especially regarding density perturbations in the conformal frame. ALB acknowledges a postdoctoral fellowship from the Japanese Society for the Promotion of Science, administered in conjunction with the National Science Foundation. KM and JY acknowledge Rocky Kolb and Theoretical Astrophysics Group for their hospitality at Fermilab. Thanks are also due to the Japan-US cooperative science program No. MPCR-185(JSPS), which supported their visit to Fermilab, where this work was completed. This work was supported in part by NASA grant \# NAGW-1340 and in part by the Grant-in-Aid for Scientific Research Fund of the Ministry of Education, Science and Culture No. 01795079. 


\section{References}

[1] A.H. Guth, Phys. Rev.D23 (1981) 347;

K. Sato, Mon. Not. Roy. Astron. Soc.195 (1981) 467;

A. Albrecht and P.J. Steinhardt, Phys. Rev. Lett. 48 (1982) 1220;

A.D. Linde, Phys. Lett. 108B (1982) 389; Phys. Lett 129B (1983) 177.

[2] R. Brandenberger, Rev. Mod. Phys. 57 (1985) 1;

A. Linde, Rep. Prog. Phys. 47 (1984) 925;

M.S. Turner, in the Proceedings of the Cargese School on Fundamental Physics and Cosmology, ed. by J. Audouze and J. Tran Thanh Van (Gif-sur-Yvette, 1985)

[3] L.A. Kofman, A.D. Linde and A.A. Starobinsky, Phys. Lett. B157 (1985) 36.

[4] A.A. Starobinsky, JETP Lett. 42 (1985) 152;

J. Silk and M. Turner, Phys. Rev. D35 (1986) 419.

[5] D. La and P.J. Steinhardt, Phys. Rev. Lett. 62 (1989) 376;

D. La, P.J. Steinhardt and E.W. Bertschinger, Phys. Lett. B231 (1989) 231.

[6] C. Brans and R.H. Dicke, Phys. Rev. 24 (1961) 925.

[7] A. Linde, CERN preprint CERN.TH.5619/89 (1989).

[8] E. Witten, Phys. Lett. 155B (1985) 151;

J.-P. Derendinger, L. Ibanez and H. Nilles, Nucl. Phys. B267 (1986) 365.

[9] G. Magnano, M. Ferraris and M. Francaviglia, Gen. Rel. Grav. 19 (1987) 465;

A. Jakubiec and J. Kijowski, Phys. Rev. D37 (1988) 1406;

K. Maeda, Phys. Rev. D39 (1989) 3159;

J.D. Barrow and K. Maeda, Waseda University preprint WU-AP/04/89 (1989).

[10] A. Zee, Phys. Rev. Lett. 42 (1979) 417.

[11] P.J.E. Peebles and B. Ratra, Astrophys. J. 325 L17;

B. Ratra and P.J.E. Peebles, Phys. Rev. D37 (1988) 3406.

[12] L.F. Abbott and M.B. Wise, Nucl. Phys. B244 (1984) 541;

F. Lucchin and S. Matarrese, Phys. Rev. D32 (1985) 1316.

[13] J.J. Halliwell, Phys. Lett. 185B (1987) 341;

J. Yokoyama and K. Maeda, Phys. Lett. 207B (1988) 31.

[14] S. Coleman and E.J. Weinberg, Phys. Rev. D7 (1973) 1888.

[15] M. Yoshimura, Phys. Rev. Lett. 41 (1978) 281;

E.W. Kolb and M.S. Turner, Ann. Rev. Nucl. Part. Sci. 33 (1983) 645.

[16] J. Yokoyama, H. Kodama, K. Sato and N. Sato, Intern. J. Mod. Phys. A 2 (1987) 1809.

[17] B.L. Spokoiny, Phys. Lett. 129B (1984) 39;

R. Fakir and W.G. Unruh, Phys. Rev. D41 (1990) 1783;

D.S. Salopek, J.R. Bond and J.M. Bardeen, Phys. Rev. D40 (1989) 1953. 
[18] R.D. Reasenberg et al, Astrophys. J. 234 (1979) L219.

[19] J. Hwang, Univ. of Texas preprint (1990).

[20] K. Sato, N. Terasawa, and J. Yokoyama, in the Proceedings of IX th Moriond Astrophysics Meeting on The Quest for the Fundamental Constants on Cosmology, ed. by J. Audouze (1990, to be published) 


\section{Figure Caption}

Fig.1 The constraints from the horizon problem, reheating temperature $\left(T_{R H}>10^{10}\right.$ $\mathrm{GeV})$ and density perturbations $\left(\delta \rho / \rho<10^{-4}\right)$ for $\beta=0.1, \psi_{0}=H_{0}$ and $\phi_{0}=10 m_{P L}$. The shaded region is permitted. The curves $R H$ and $D$ are the constraints from the reheating temperature and density perturbations, respectively. The point + corresponds to the $\mathrm{SU}(5)$ model with $V_{0}^{1 / 4}=10^{15} \mathrm{GeV}$ and $\lambda \sim 1 / 2$. The dotted line $\mathrm{RH}_{\mathrm{JBD}}$ shows the reheating constraint for the JBD theory with $\omega \sim 500$. 


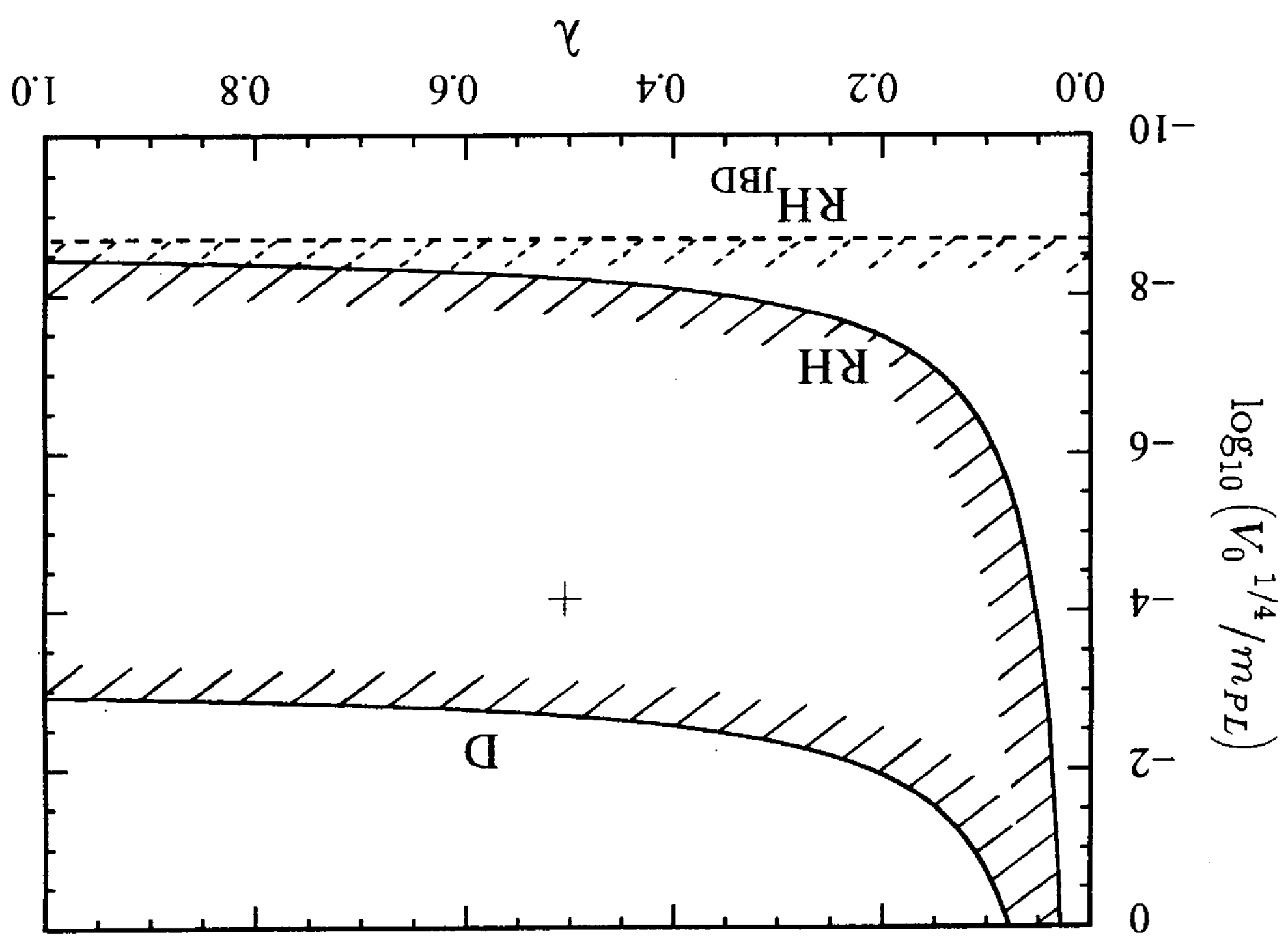

I'8! 